\title{
Automatic Verification of Pointer Data-Structure Systems for All Numbers of Processes ${ }^{\star}$
}

\author{
Farn Wang \\ Institute of Information Science, Academia Sinica \\ Taipei, Taiwan 115, Republic of China \\ +886-2-27883799 ext. 1717; FAX +886-2-7824814; \\ farn@iis.sinica.edu.tw
}

\begin{abstract}
Real-world concurrent software may be implemented with any number of processes which are linked together to form complex and dynamic network configurations. We formally model such concurrent software as processes running algorithms on data-structures with pointers. We show that the verification problem of such algorithms is undecidable. A new automatic approximation method is then proposed to safely verify such algorithms. The central idea is to construct a finite collective image set $(C I S)$ which collapses reachable state representations for all implementations of all numbers of processes. Our collapsing scheme filters out unimportant information of system behaviors and results in CIS's with manageable space requirements. Analysis shows our method can automatically generate a CIS of size 1619 to verify that a version of Mellor-Crummy \& Scott's algorithm preserves mutual exclusion for all numbers of processes.
\end{abstract}

\section{Introduction}

With the success of automatic verification technology for hardware systems in recent years 6] 8, people are now naturally looking forward to automating the tasks of software verification. However, with vast variety of abstract devices like unlimited concurrencies, pointers, dynamic data-structures, range-unbounded variables, unbounded buffers, ......, etc., software systems are far more sophisticate than hardware systems. Straightforward extension of the existing statebased technology 4, 9, 10, 13, 19, 21, 24] is at an inappropriate abstractness level and has generally bumped into steep complexities of the verification problems for software systems 1, 2, 14, 22, 23. In this paper, we propose a formal system to model such concurrent softwares and show that in general the corresponding verification problem is undecidable. Then we present a new automatic approximation verification method which reasons at an abstractness level similar to that

\footnotetext{
* The work is partially supported by NSC, Taiwan, ROC under grant NSC 88-2213E-001-002.
} 


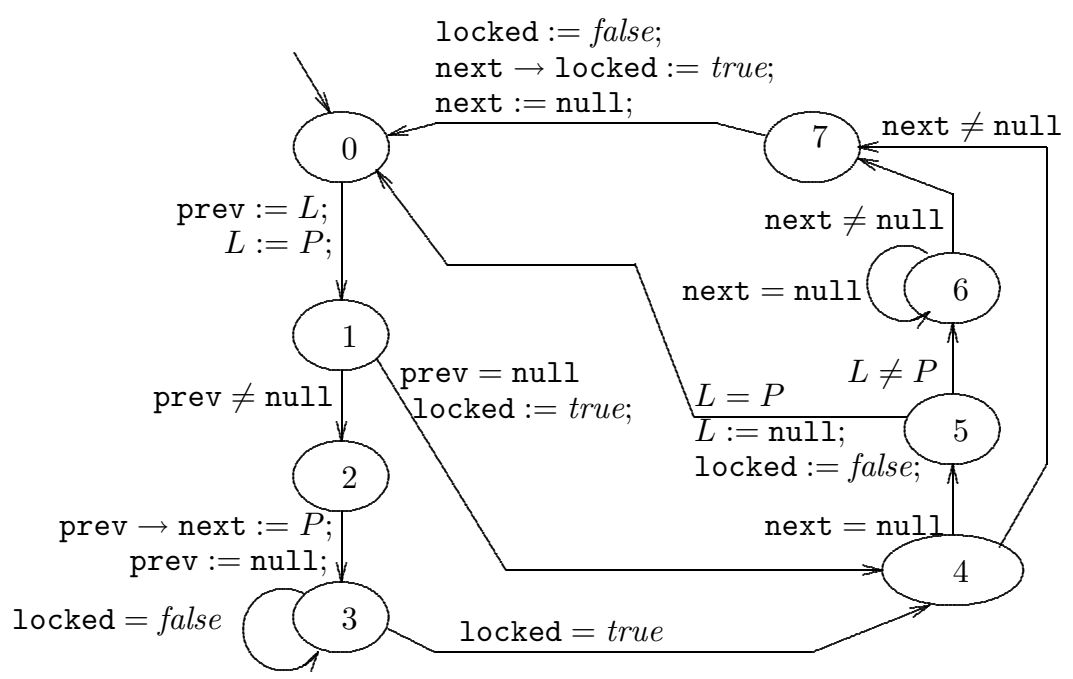

Fig. 1. modified MCS locking algorithm

of humans by filtering out unimportant details of system behaviors for efficient veification.

We are dealing with concurrent systems of unspecified number of processes running different copies of a same algorithm with data-structures of enumerate variables and pointers which are either global or local. With such pointers, the processes can dynamically compose sophisticate network configurations.

Example 1. : Mellor-Crummy \& Scott's algorithm MCS locking algorithm[18 is an example protocol in which a global waiting queue of processes is explicitly used to insure mutual exclusion to the critical section in a concurent system. In figure 1 a modified version of MCS locking algorithm for a process is drawn, as a finite-state automaton, while the original version is given in figure 5 in the appendix. The critical section is mode 4 . The queue is constructed with one global pointer L to the tail of the queue, and each process's local pointers: next and prev which respectively point to the successor and predecessor processes of the local process in the queue. $P$ is the special symbol for the address of the running process. Each process also has one Boolean variable locked which is set to true when the process can enter the critical section. A process can access and manipulate other processes' information through global and local pointers.

MCS algorithm maintains mutual exclusion by insuring that at any moment at most one process has its local locked set to true and the process sets its waiting queue successor's locked to true after it changes its own locked to false. 
In section 3 we shall prove that the verification problem of such algorithms is undecidable, i.e. no computers with finite memory can answer such a queustion. Since the verification problem is extremely difficult, we instead develop an automatic approximation method which can answer the safety of a large class of such algorithms regardless of the number of processes. The idea is to construct a finite collective image set (CIS) whose elements are reachable state images describing the behaviors of all implementations with any number of processes. Engineers' intelligence and experiences in design and verification is encoded in the mapping from states to images in CIS's and seems to result in small CIS's even for complicate data-structures. For safety analysis, if we can construct a finite CIS which contains no images of states violating the safety specification, then it is good enough to conclude that the algorithm is safe for any number of processes. However if there is a state image violating the safety specification in the CIS, then no conclusion can be made because the image may be included due to insufficient approximation precision.

With the known high complexities of most verification problem models [1, 2, $14,22,23$, it is clear that current technology cannot identify a large class of concurrent algorithms subject to efficient verification. On the other hand, we argue that our technology can serve to identify such a large class of "well-designed" concurrent algorithms which can be efficiently verified. In section 5 we shall establish the mighty lemma 3 which allows us to eliminate much combinatorial complexity in CIS without sacrifising approximation precision. In section 6, we shall analyze our method on the modified MCS algorithm (in example1) in which local pointers are set to null whenever the current values of the pointers will not be used in the future. The modification is consistent with good programming practice of elimination of "stray" pointers. The interesting thing here is that our method can generate a small CIS of size 1619 for the modified MCS algorithm while fails to do so for the original one. This shows that our verification method is indeed more efficient for "good" designs.

We shall adopt the following notations. Given a set or sequence $K,|K|$ is the number of elements in $K$. For each element $e$ in $K$, we also write $e \in K$. We let $\mathcal{N}$ be the set of nonnegative integers.

\section{Related Work}

Apt and Kozen already showed that in general verification of systems with unknown number of concurrent processes is undecidable 3. This means that such verification problems are extremely hard and we can only rely on semi-decision procedures or, as in this work, approximation algorithms to answer them. Otherwise, we can also investigate to find out decidable subclasses of the problem. In the following, we briefly describe some of the related work.

Browne, Clarke, and Grumberg [5] use bisimulation equivalence relation between global state graphs of systems of different sizes. The equivalence relation must be strong enough for the method to work. Thus the construction of the equivalence relation is difficult to mechanize. 
Clarke, Grumberg, and Jha[11] propose to use regular languages to specify properties in a linear network with unknown number of processes. Then stateequivalence relation is defined based on the regular languages and a mechanical method is defined to synthesize a network invariant $\mathcal{I}$ in the hope that $\mathcal{I}$ can be contained by the specification. But there is no guarrantee that $\mathcal{I}$ is a model of the specification even if the system indeed satisfies the specification. Moreover, it is not known whether using the specification regular languages to derive equivalence class properly perserves the reasonings behind the system design. Lesens, Halbwachs, and Raymond 17 furthered the approach by designing a language for the specification in systems with complex structures and by using fixed-point resolution with different heuristics to calculate many network invariants. Compared to our approach, we argue that the technique of CIS better captures the design reasoning that the relations between processes in different states are far more important than the actual numbers of processes in different states. We believe in verifying complex systems, without utilizing the reasoning behind the system designs, state-explosion problem cannot be properly dealt with.

Kurshan and McMillan[16] proposes to use network structural induction which is not guarranteed to terminate. Also inductive hypothesis is difficult to construct, although once it is ready, the whole approach is usually very efficient. Compared to our approach, we are using an approximation algorithm which captures the engineers' view of linear list. Users only have to guess the value of bound $B$, used in CIS construction, which for many real-world concurrent algorithms, small value like 1 will do.

Emerson and Naamjoshi[12] specialized on static token ring networks. They prove that for certain properties, verification on small size networks can be used to guarrantee the verification of large size networks. In contrast, our method is applicable to all different configurations of "dynamic" networks of processes.

Boigelot and Godefroid[7] choose to use state-space exploration to handle the verification problems of systems with unbounded FIFO queues. Their statespace representation is constructed by collapsing FIFO queues. Their approach does not guarrantee termination.

Recently, the author also has researched on the technique of collective quotient structures on dynamic linear networks [20]. The idea is similar to that of CIS in that they both collapse state-spaces of all implementations into single structures. However my work here is more general for pointer data-structures which allows the development of lemma 3, in section 5, and can lead to significant reduction in time and space complexity.

\section{Concurrent Algorithms and Safety Bound Problem}

We are dealing with concurrent algorithms with a local data structure for each process. The address of a data structure can be viewd as the identity of the corresponding process. We shall have the convention that if a process is named $p$, then $p$ is also the address of process $p$ 's data-structure. 
Two types of variables can be declared. The first is the type of enumerate variables with predefined finite integer value ranges. For convenience, we can also give symbolic names to those integer values. Traditionally, false is interpreted as 0 while true as 1 . The second is the type of pointers (address variables) to processes (data-stuctures). As in example $1 L$ is a pointer to the tail of a queue. Variables can be declared as global variables which all processes can access, or local variables of a process which only the declaring process can directly access. The same name can be used to represent the respective local variables of different processes. For example, in example 1 different processes access different variables which are all locally called locked.

Test can be made to determine if an enumerate type variable's content equals to a constant, if a pointer is null, or if two pointers point to the same process. We can also assign a constant to an enumerate type variable or to assign a process address to a pointer. In the following, we shall first formally define the syntax and semantics of our systems, and then define the safety bound problem.

\subsection{Syntax of Algorithm Descriptions}

Conceptually, a concurrent algorithm $S$ is a tuple $\left(\mathrm{Enu}_{g}, \mathrm{Ptr}_{g}, \mathrm{Enu}_{l}, \mathrm{Ptr}_{l}, A(P)\right)$ where $\mathrm{Enu}_{g}$ and $\mathrm{Enu}_{l}$ are respectively the sets of global and local enumerate variables (as in Pascal programming language), $\mathrm{Ptr}_{g}$ and $\mathrm{Ptr}_{l}$ are respectively the sets of global and local pointers, and $A(P)$ is the process program template, with process identifier symbol $P$.

Given algorithm $S=\left(\mathrm{Enu}_{g}, \mathrm{Ptr}_{g}, \mathrm{Enu}_{l}, \mathrm{Ptr}_{l}, A(P)\right)$, a process predicate pred of $S$ can be used to describe the triggering condition of state transitions and has the following syntax.

$$
\begin{aligned}
& \operatorname{pred}::=\exp _{1}=\exp _{2} \mid \text { ref }_{1}=\text { ref }_{2} \mid \neg \text { pred } \mid \text { pred }_{1} \vee \text { pred }_{2} \\
& \exp ::=c|x| z|y \rightarrow x| w \rightarrow x \\
& \text { ref }::=\operatorname{null}|P| w|y| w \rightarrow y \mid y_{1} \rightarrow y_{2}
\end{aligned}
$$

where $c \in \mathcal{N}, x \in \mathrm{Enu}_{l}, z \in \mathrm{Enu}_{g}, w \in \mathrm{Ptr}_{g}$, and $y \in \operatorname{Ptr}_{l}$. Traditional shorthands are $\exp _{1} \neq \exp _{2} \equiv \neg\left(\exp _{1}=\exp _{2}\right)$, ref $_{1} \neq \operatorname{ref}_{2} \equiv \neg\left(\operatorname{ref}_{1}=\operatorname{ref}_{2}\right)$, $\operatorname{pred}_{1} \wedge \operatorname{pred}_{2} \equiv \neg\left(\left(\neg\right.\right.$ pred $\left.\left._{1}\right) \vee\left(\neg \operatorname{pred}_{2}\right)\right)$, and pred $_{1} \Rightarrow$ pred $_{2} \equiv\left(\neg\right.$ pred $\left._{1}\right) \vee$ pred $_{2}$, Thus a process may operate on conditions of the global and local variables, and also on the local variables of the processes pointed to by global pointers. We let Predicate $_{S}$ be the set of all process predicates of $S$.

Once the triggering condition is satisfied by a running process, the process may execute a finite sequence, say actseq, of actions to update the state information. The finite sequence actseq of actions has the following syntax.

$$
\begin{aligned}
& \operatorname{actseq}::=\mid \text { act actseq } \\
& \text { act }::=\text { lhsexp }:=\exp ; \mid \text { lhsref }:=\text { ref; } \\
& \text { lhsexp }::=x|z| w \rightarrow x \mid y \rightarrow x \\
& \text { lhsref }::=y|w| w \rightarrow y \mid y_{1} \rightarrow y_{2}
\end{aligned}
$$


Here exp and ref are defined as in the syntax of process predicates. act defines what an action looks like. The set of all finite sequences of actions of $S$ is named Aseqset $_{S}$.

Given a concurrent algorithm $S=\left(\mathrm{Enu}_{g}, \mathrm{Ptr}_{g}, \mathrm{Enu}_{l}, \mathrm{Ptr}_{l}, A(P)\right), A(P)$ is the program template for each process with identifier symbol $P$. Program template $A(P)$ has the syntax similar to that of finite-state automata. $A(P)$ is conceptually a tuple $\left(Q, q_{0}, E, \tau, \pi\right)$ with the following restrictions.

- $Q$ is a finite set of operation modes.

- $q_{0} \in Q$ is the initial operation mode.

- $E \subseteq Q \times Q$ is the set of transitions among operation modes.

- $\tau: E \mapsto$ Predicate $_{S}$ is a mapping which defines the triggering condition of each transition.

- $\pi: E \mapsto$ Aseqset $_{S}$ is a mapping which defines the action sequence performed at the happening of each transition. Atomicity of the transition is assumed. We do require that there is a variable mode $\in \mathrm{Enu}_{l}$ which records the current operation mode of the corresponding process. However, while drawing $A(P)$ as an automaton, we omit the description of mode values in the triggering conditions and action sequences for simplicity and clarity.

\subsection{Computation of Systems}

Let $\Pi$ be the set of all processes (conceptually represented by either their identifiers, or their data-structure addresses) in an implementation. For each enumerate-type variable $x$, we let $D_{x}$ be $\{0\}$ unioned with the set of all constants assigned to $x$ in process program $A(P)$ labeled in all transition's assignment sequence. Especially, $D_{\text {mode }}=Q$ and mode $=0$ means the process is in its initial operation mode.

A process state $\pi$ is a mapping from $\mathrm{Enu}_{l} \cup \operatorname{Ptr}_{l}$ to $\mathcal{N} \cup \Pi \cup\{$ null $\}$ such that $\pi(x) \in D_{x}$ if $x \in \mathrm{Enu}_{l}$; and $\pi(x) \in \Pi \cup\{$ null $\}$ if $x \in \operatorname{Ptr}_{l}$.

A global state of $S \Pi$ is a pair $(\psi, \phi)$ with the following restrictions.

- $\psi$ is a mapping from $\mathrm{Enu}_{g} \cup \operatorname{Ptr}_{g}$ to $\mathcal{N} \cup \Pi \cup\{$ null $\}$ such that $\psi(x) \in D_{x}$ if $x \in \mathrm{Enu}_{g}$; and $\psi(x) \in \Pi \cup\{$ null $\}$ if $x \in \operatorname{Ptr}_{g}$.

- $\phi$ is a mapping from $\Pi$ to the set of all process states running algorithm $A$. Given a global state $\nu=(\psi, \phi)$, a process $p \in \Pi$, and a process predicate pred $\in$ Predicate $_{S}$, we define the relation of $p$ satisfies pred at $\nu$, written $p, \nu \models$ pred, in the following inductive way. Assume that $x \in \mathrm{Enu}_{l}, z \in \mathrm{Enu}_{g}, y, y_{1}, y_{2} \in \mathrm{Ptr}_{l}$, and $w \in \operatorname{Ptr}_{g}$.

- $p, \nu \models \exp _{1}=\exp _{2}$ iff value $\left(p, \nu, \exp _{1}\right)=\operatorname{value}\left(p, \nu, \exp _{2}\right)$

- $\operatorname{value}(p, \nu, c)=c$

- $\operatorname{value}(p, \nu, x)=\phi(p)(x)$

- $\operatorname{value}(p, \nu, z)=\psi(z)$

- $\operatorname{value}(p, \nu, y \rightarrow x)=\phi(\phi(p)(y))(x)$

- $\operatorname{value}(p, \nu, w \rightarrow x)=\phi(\psi(w))(x)$

- $p, \nu \models r e f_{1}=\operatorname{ref}_{2}$ iff value $\left(p, \nu, \operatorname{ref}_{1}\right)=\operatorname{value}\left(p, \nu, r e f_{2}\right)$

- $\operatorname{value}(p, \nu$, null $)=$ null

- $\operatorname{value}(p, \nu, P)=p$ 
- $\operatorname{value}(p, \nu, w)=\psi(w)$

- $\operatorname{value}(p, \nu, y)=\phi(p)(y)$

- $\operatorname{value}(p, \nu, w \rightarrow y)=\phi(\psi(w))(y)$

- $\operatorname{value}\left(p, \nu, y_{1} \rightarrow y_{2}\right)=\phi\left(\phi(p)\left(y_{1}\right)\right)\left(y_{2}\right)$

- $p, \nu \models \neg$ pred iff it is not the case that $p, \nu \models$ pred

- $p, \nu=\operatorname{pred}_{1} \vee \operatorname{pred}_{2}$ iff $p, \nu \models \operatorname{pred}_{1}$ or $p, \nu \models \operatorname{pred}_{2}$

Given an action act of $S$, the new global state obtained by applying act to $p$ at $\nu$, written next_state $(p, \nu$, act $)$, is defined in the following way.

- $\left(\psi^{\prime}, \phi^{\prime}\right)=$ next_state $(p, \nu, x:=\exp ;)$ is identical to $\nu$ except that $\phi^{\prime}(p)(x)=\operatorname{value}(p, \nu, \exp )$.

- $\left(\psi^{\prime}, \phi^{\prime}\right)=$ next_state $(p, \nu, z:=\exp ;)$ is identical to $\nu$ except that $\psi^{\prime}(z)=\operatorname{value}(p, \nu, \exp )$.

- $\left(\psi^{\prime}, \phi^{\prime}\right)=$ next_state $(p, \nu, w \rightarrow x:=\exp ;)$ is identical to $\nu$ except that $\phi^{\prime}(\psi(w))(x)=\operatorname{value}(p, \nu, \exp )$.

- $\left(\psi^{\prime}, \phi^{\prime}\right)=$ next_state $(p, \nu, y \rightarrow x:=\exp ;)$ is identical to $\nu$ except that $\phi^{\prime}(\phi(p)(y))(x)=\operatorname{value}(p, \nu, \exp )$.

- $\left(\psi^{\prime}, \phi^{\prime}\right)=$ next_state $(p, \nu, y:=$ ref; $)$ is identical to $\nu$ except that $\phi^{\prime}(p)(y)=\operatorname{value}(p, \nu$, ref $)$.

- $\left(\psi^{\prime}, \phi^{\prime}\right)=$ next_state $(p, \nu, w:=$ ref; $)$ is identical to $\nu$ except that $\psi^{\prime}(w)=\operatorname{value}(p, \nu$, ref $)$.

- $\left(\psi^{\prime}, \phi^{\prime}\right)=$ next_state $(p, \nu, w \rightarrow y:=$ ref; $)$ is identical to $\nu$ except that $\phi^{\prime}(\psi(w))(y)=\operatorname{value}(p, \nu, r e f)$.

- $\left(\psi^{\prime}, \phi^{\prime}\right)=$ next_state $\left(p, \nu, y_{1} \rightarrow y_{2}:=\right.$ ref; $)$ is identical to $\nu$ except that $\phi^{\prime}\left(\phi(p)\left(y_{1}\right)\right)\left(y_{2}\right)=\operatorname{value}(p, \nu$, ref $)$.

Given an action sequence $\operatorname{act}_{1} \ldots \operatorname{act}_{n} \in \operatorname{Aseqset}_{S}$, we let next_state $(p, \nu$,

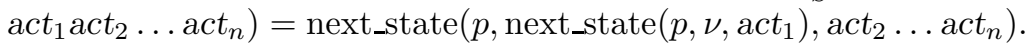

The initial state $\left(\psi_{0}, \phi_{0}\right)$ of an implementation $S \Pi$ must satisfies the following restrictions: (1) $\psi_{0}(z)=0$ for all $z \in \mathrm{Enu}_{g},(2) \psi_{0}(w)=$ null for all $w \in \operatorname{Ptr}_{g},(3) \phi_{0}(p)(x)=0$ for all $p \in \Pi$ and $x \in \mathrm{Enu}_{l}$, and $(4) \phi_{0}(p)(y)=$ null for all $p \in \Pi$ and $y \in \operatorname{Ptr}_{l}$. We assume that processes interact with interleaving semantics in the granularity of transitions, that is at any moment, at most one process can execute a transition. Interleaving semantics is well-accepted in verification theory for its simplicity. From the viewpoint of languages, such an atomicity may be difficult to implement. Since our focus is on verification and modelling, instead of implementation, we believe such an atomicity is important in modelling embedded systems in which natural world atomic transitions can only be emulated by several algorithm actions.

A computation of an implementation $S \Pi$ is a (finte or infinite) sequence $\rho=\nu_{0} \nu_{1} \ldots \nu_{k} \ldots \ldots$ of global states with $\nu_{k}=\left(\psi_{k}, \phi_{k}\right)$ for all $k \geq 0$ such that

- $\nu_{0}$ is the initial state of $S \Pi$; and

- for each $\nu_{k}$ with $k>0$, there is a $p \in \Pi$ and transition from $q$ to $q^{\prime}$ such that $p, \nu_{k-1} \models \tau\left(q, q^{\prime}\right)$ and next_state $\left(p, \nu_{k-1}, \pi\left(q, q^{\prime}\right)\right)=\nu_{k}$. 


\subsection{Safety Bound Problem and Its Undecidability}

The computation definition of our algorithm implementations is independent of the real names used for each process in $\Pi$. Never the names of processes are used to affect the behaviors of our implementations. Instead, only the relation among processes in various process states is important. Thus it is better if we can present our safety analysis problem regardless of the actual names used for processes. Given a global state $\nu=(\psi, \phi)$ of an implementation $S \Pi$ and a process predicate pred, $\operatorname{count}_{\text {pred }}(\nu)$ is the number of processes satisfying pred at $\nu$, i.e. $\mid\{p \mid p \in \Pi ; p, \nu \models$ pred $\} \mid$. A computation $\rho=\nu_{0} \nu_{1} \ldots \nu_{k} \ldots \ldots$ of $S \Pi$ violates safety property pred with bound $c \in \mathcal{N}$ iff there is a $k \geq 0$ such that count $_{\text {pred }}\left(\nu_{k}\right)>c$.

The safety bound problem instance $\operatorname{SBP}(S$, pred,c) is to determine if for all finite sets $\Pi$ of processes and all computation $\rho$ of $S \Pi, \rho$ does not violate safety property pred with bound $c$. Such a problem framework can be used to verify process state reachability problem[14] which is a special case of $\operatorname{SBP}(S$, pred,c) with $c=1$. Also mutual exclusion problem can be formulated with $c=1$. Reader-Writer problem can be formulated with $c$ set to the number of readers.

Example 2. : Consider the modified MCS algorithm in example 1. The critical section is in mode 4 . A process can enter the critical section only if its local variable locked is true. Thus the safety bound problem for the mutual exclusion to critical section can be formulated as $\operatorname{SBP}(S$, locked $=$ true, 1$)$ which answers "true" iff along any computation $\rho$ of all implementations, no more than one process can have locked $=$ true simultaneously.

However, such a problem is extremely difficult to answer. In fact, we can show $\operatorname{SBP}(S$, pred, 1$)$ for a given $S$ and pred is undecidable, i.e. there is no computer with finite amount of memories capable of answering $\operatorname{SBP}(S$, pred, 1$)$. Lemma 1 proves this by reducing two-counter machine halting problem[15] to $\operatorname{SBP}(S$, pred, 1$)$. A two-counter machine $M$ has a finite-state control and two counters which can hold any natural numbers. The finite-state control can increment a counter, decrement a counter, or transit between finitely many operation modes by testing whether a particular counter contains zero. It is known that two-counter machine can emulate Turing machine whose halting problem cannot be answered by any computers with finite amount of memories.

Lemma 1.: Two-counter machine halting problem is reducible to $\operatorname{SBP}(S$, pred, 1).

Proof : Due to page-limit, we shall only give a sketch of the proof. Suppose we are given a two-counter machine $M$. We want to show that we can construct a concurrent algorithm $S$ such that $M$ reaches its final state iff there is an implementation $S \Pi$ such that there is a process in $\Pi$ which also reaches its corresponding local final state. We shall implement two stacks to emulate the two counters respectively with pointers linking together adjacent elements in the stacks. The first (second) stack's height of $S \Pi$ emulates the content of the first (second) counter of $M$. Suppose in the computation of $M$ to reach its 
final state, counter 1 and 2 have maximum contents $v_{1}$ and $v_{2}$ respectively. Then there exists a $\Pi$, of $3+v_{1}+v_{2}$ processes, which makes the construction works.

The halting state of $M$ is encoded in pred. The first transiting process in the computation will be used to emulate the finite-state control. The second and third transiting processes in the computation will respectively be used to emulate the stack bottoms for the two counters. Then each increment operation of a counter will need one process to be pushed onto the corresponding stacks. If there is not enough number of processes for the increment operations in the implementation, then the computation simply halts in a state without satisfying pred. Each decrement operation of a counter will need the top process in the corresponding stack to be popped. Testing for zero value of a counter can be implemented by asking if the stack top process is equal to the stack bottom process for the corresponding counter. In this way, we can construct $S$ and pred such that $\operatorname{SBP}(S$, pred, 1$)$ answers true iff $M$ reaches its halting state.

\section{Collective Image Set}

With lemma 1 and many similar complexity results [1, 2, 14, 22, 23, it is clear that classic verification technology is not able to handle the complexity incurred by verification problems for concurrent algorithms with sophisticate datastructures. However, we have observed that classic verification theory does not distinguish "well-behaved" systems from "bad" systems. In many algorithms for concurrent systems, the number of processes is usually not a crucial factor in the correctness of systems.

Our CIS is a set whose elements are global state images which are finite recordings of multisets of process information patterns. A multiset is conceptually a set which allows an element to repeat many times. Mathematically, it is a mapping from a domain to $\mathcal{N}$. We say that process $p$ points to process $p^{\prime}$ (and $p^{\prime}$ is called a reference of $\left.p\right)$ in state $(\psi, \phi)$ if there is either a $y \in \operatorname{Ptr}_{l}$ such that $\phi(p)(y)=p^{\prime}$ or a $w \in \operatorname{Ptr}_{g}$ such that $\psi(w)=p^{\prime}$. State of each process $p$ is collapsed down to a PDSI (process data-structure image) which only records information that process $p$ can read from the local variables of itself and its global or local references.

A global state image, called GDSI (global data-structure image), is treated as a finite recording of a multiset of PDSI's of the participating processes. It is finite because when more than $B$, a constant chosen by users, processes have the same PDSI in a global state, they are only recorded by a flag ( $\infty$ here) which denotes that the number of processes in that PDSI exceeds $B$. By viewing the GDSI's as a multiset, we treat all processes in a symmetric way without the price for the management of process identifier permutation [13. With the constant $B$, we are able to map states of infinitely many implememtations down to finitely many global state images. To choose a value for $B$, users should have the intuition that when more than $B$ processes are of a PDSI, the actual number of such processes is not important. For example, for many mutual exclusion algorithms, if there are either 3 or 4 processes in the waiting queue, the processes in each PDSI's 
exhibit the same behavior pattern. For example, to pass over the right to the critical section, the process in the critical section (of one PDSI) passes the right to the head process in the waiting queue (of another PDSI). The behavior pattern only depends on whether there is a process in the waiting queue. According to our observation, many algorithms work with such reasoning. For a lot of mutual exclusion protocols, small value of $B$ like 1 will work and the CIS's exhibit simple regularity.

In the following subsections, we shall define rigorously the image mapping of global states of implementations. Then we shall define the transitions, among GDSI's, which corresponds to transition rules described in $A(P)$.

We need the following conventions regarding number systems respecting a bound $B$. Let $\mathcal{N}^{\infty}=\mathcal{N} \cup\{\infty\}$ where $\infty$ means any number greater than $B$. For any $c \in \mathcal{N}, c<\infty$. For any $c, d \in \mathcal{N}^{\infty}, c \leq \infty$ and $c+\infty=\infty+d=\infty$.

Given any two numbers $c$ and $B$ in $\mathcal{N}^{\infty}$, we let $c^{(B)}=c$ if $c \leq B ; c^{(B)}=\infty$ if $c>B$. Finally, $[0, B]^{(\infty)}=\{0,1, \ldots, B\} \cup\{\infty\}$.

\subsection{Pointer Data-Structure Images}

The global-state images in our method is characterized by finite sets of propositional atoms. We shall first define PSI (process state images) as building blocks to construct PDSI. PSI represents the observation a process can make without going through pointers. The PSI of process $p$ at state $\nu=(\psi, \phi)$, in symbols $\operatorname{PSI}(p, \nu)$, is a finite set of atoms constructed in the following way.

$$
\begin{aligned}
&\left\{x=c \mid x \in \operatorname{Enu}_{l} ; c \in D_{x} ; c=\phi(p)(x)\right\} \\
& \cup\left\{z=c \mid z \in \operatorname{Enu}_{g} ; c \in D_{x} ; c=\psi(z)\right\} \\
& \cup\left.\cup P=y \mid y \in \operatorname{Ptr}_{l} ; p=\phi(p)(y)\right\} \\
& \cup\left.\cup P=w \mid w \in \operatorname{Ptr}_{g} ; p=\psi(w)\right\} \\
& \operatorname{PSI}(p, \nu)= \cup\left\{y=\operatorname{null} \mid y \in \operatorname{Ptr}_{l} ; \phi(p)(y)=\operatorname{null}\right\} \\
& \cup\left\{w=\operatorname{null} \mid w \in \operatorname{Ptr}_{g} ; \psi(w)=\operatorname{null}\right\} \\
& \cup\left\{y=w \mid y \in \operatorname{Ptr}_{l} ; w \in \operatorname{Ptr}_{g} ; \phi(p)(y)=\psi(w)\right\} \\
& \cup\left\{y_{1}=y_{2} \mid y_{1}, y_{2} \in \operatorname{Ptr}_{l} ; \phi(p)\left(y_{1}\right)=\phi(p)\left(y_{2}\right)\right\} \\
& \cup\left\{w_{1}=w_{2} \mid w_{1}, w_{2} \in \operatorname{Ptr}_{g} ; \phi(p)\left(w_{1}\right)=\phi(p)\left(w_{2}\right)\right\}
\end{aligned}
$$

Here we use $P$ to symbolically represent the address $p$ of the corresponding process. Note that we conveniently define PSI's to also record information on global variables. Thus in our GDSI defined later, PSI's of all processes must all agree on the informations of those global variables. Conveniently, we shall let PSIset be the set of all PSI's.

Our process image, the PDSI (process data-structure image (PDSI)) of a process $p$ in a state $\nu=(\psi, \phi)$ with bound $B$, in symbols $\operatorname{PDSI}^{(B)}(p, \nu)$, is graphically shown in figure 2 and only records

- the PSI's of $p$ and $p$ 's global and local references;

- the equality among $p$ and $p$ 's references' references (i.e. if the references point back); and

- the multiset of incoming local pointers from peer processes to $p$ with bound $B\left(\operatorname{ILM}^{(B)}\right.$ for incoming link multiset with bound $B$, will be defined later). 


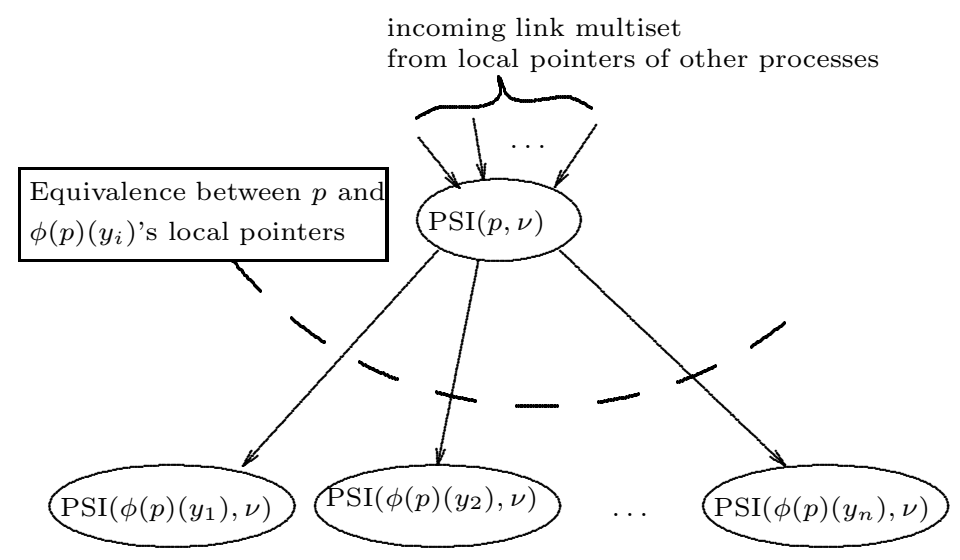

Fig. 2. Information of PDSI

Intuitively, PDSI of a process $p$ records the information that $p$ can infer by at most one dereferencing through its local and global pointers. In the following, we shall make the definition of PDSI more precise.

The incoming link multiset (ILM) of process $p$ at state $\nu=(\psi, \phi)$, in symbols $\operatorname{ILM}(p, \nu)$, records those peer processes which have $p$ as their global or local references. When a transiting process $p$ changes the PSI of itself and its references, the PDSI's of processes with $p$ as their references will also be changed. To propagate such waves of PDSI changes, we need information in $\operatorname{ILM}(p, \nu)$ to enumerate the possibilities. Formally speaking, for all $\lambda \in$ PSIset and $y \in \operatorname{Ptr}_{l}$ in an algorithm implementation $S \Pi$,

$$
\operatorname{ILM}(p, \nu)(\lambda, y)=\left|\left\{\left(p^{\prime}, y\right) \mid p^{\prime} \in \Pi ; \operatorname{PSI}\left(p^{\prime}, \nu\right)=\lambda ; \phi\left(p^{\prime}\right)(y)=p\right\}\right|
$$

To respect bound $B$, we let $(\operatorname{ILM}(p, \nu))^{(B)}$ be a mapping from the domain to $[0, B]^{(\infty)}$ such that for all $\lambda \in$ PSIset and $y \in \operatorname{Ptr}_{l},(\operatorname{ILM}(p, \nu))^{(B)}(\lambda, y)=$ $(\operatorname{ILM}(p, \nu)(\lambda, y))^{(B)}$.

Given an algorithm, it is clear that the number of different possible PDSI's at states of all implementations is finite. We let PDSIset ${ }^{(B)}$ be the set of all possible PDSI's with bound $B$.

The global data-structure image (GDSI) $\chi$ of a state $\nu=(\psi, \phi)$ with bound $B$, in symbols $\operatorname{GDSI}^{(B)}(\nu)$, is a mapping from $\operatorname{PDSIset}^{(B)}$ to $[0, B]^{(\infty)}$ such that for all $\mu \in \operatorname{PDSIset}^{(B)}$, if $\operatorname{GDSI}^{(B)}(\nu)(\mu) \neq \infty$, it means that there are exactly GDSI ${ }^{(B)}(\nu)(\mu)$ processes in $\nu$ whose PDSI's are $\mu$; otherwise, it means that there are more than $B$ processes in $\nu$ whose PDSI's are $\mu$. Since GDSI's are constructed with finite set of atomic propositions and constant $B$, it is clear that the number of GDSI's is finite.

Notationally, we let GDSIset ${ }^{(B)}$ be the set of all distinct GDSI's with $B$. 


\subsection{Transitions among GDSI's}

We let $\mathbf{x t i o n}_{e}\left(\chi, \chi^{\prime}\right)$ be the relation, between $\chi$ and $\chi^{\prime}$, which is true iff from GDSI $\chi$, we can transit to GDSI $\chi^{\prime}$ through transition rule $e$. $\operatorname{xtion}_{e}\left(\chi, \chi^{\prime}\right)$ can be computed by the following nondeterministic procedure.

A. Nondeterministically choose PDSI's in $\chi$ corresponding to the transiting process and its references.

B. If they are inconsistent, then answer false and stop.

C. Change the PSI's and PDSI's of those chosen PDSI's according to the meaning of transition rule $e$.

D. Nondeterministically propagate the changes to other unchosen PDSI's according to the ILM recordings of those chosen PDSI's.

E. After all propagations are done, if $\chi$ is changed to $\chi^{\prime}$, answer true; else answer false.

The steps are written in nondeterministic style for simplicity. If and only if one of the executions end up with return value true, then $\chi$ can go to $\chi^{\prime}$ with transition rule $e$. In practice, they have to be implemented with recursive procedure-calls. In each level of the recursion, a choice is made and recorded in the recursion stack.

We shall demonstrate the working of the above-mentioned procedure for the transition rule from mode 0 in the modified MCS algorithm (figure 1). The rule in guarded command language is

$$
\text { mode }=0 \rightarrow(\text { mode }:=1 ; \text { prev }:=L ; L:=P ;)
$$

In step $\mathrm{A}$, in case we choose $\mu \in \operatorname{PDSIset}^{(B)}$, with $\chi(\mu) \neq 0$, for either the transiting process or one of its references, what we actually do is to create a new PDSI $\mu^{\prime}$ in $\chi$ with $\chi\left(\mu^{\prime}\right)=1$ to represent the chosen process. Then we have to decrement the value of $\chi(\mu)$ respecting bound $B$. That is, if $\chi(\mu) \neq \infty$, then the decremented value can either be $\infty$ or $B$ nondeterministically; otherwise, the decremented value is $\chi(\mu)-1$.

Suppose that we pick $\mu_{P}$ as the transiting process with $\chi\left(\mu_{P}\right)=\infty$. According to our modification to MCS algorithm, the two local pointers: prev and next of $\mu_{P}$ both point to null. For convenience, we shall adopt notations like $\mu_{P} \rightarrow$ prev for the PDSI which represents the reference prev of PDSI $\mu_{P}$ in a GDSI. $\mu_{P} \rightarrow$ mode also has the same intuition. The only other reference which $\mu_{P}$ can make is through global pointer $L$. Suppose that we pick $\mu_{L}$ with $\mu_{L} \rightarrow \operatorname{mode}=2$ as the global reference $L$. It can be shown from our CIS construction that $\chi\left(\mu_{L}\right)=1$. This is natural because a global pointer can only point to one process. Thus after step A, we have the following pictorial description of those chosen PDSI's.

Note since our modification to MCS algorithm eliminates strayed pointers (see appendix), $\mu_{L} \rightarrow$ prev, if not null, will be unique in $\chi$, i.e., $\chi\left(\mu_{L} \rightarrow\right.$ prev $)=$ 1. Suppose after a nondeterministic choice of $\mu_{P}$ and $\mu_{L}, \chi$ is changed to $\chi_{A}$. 

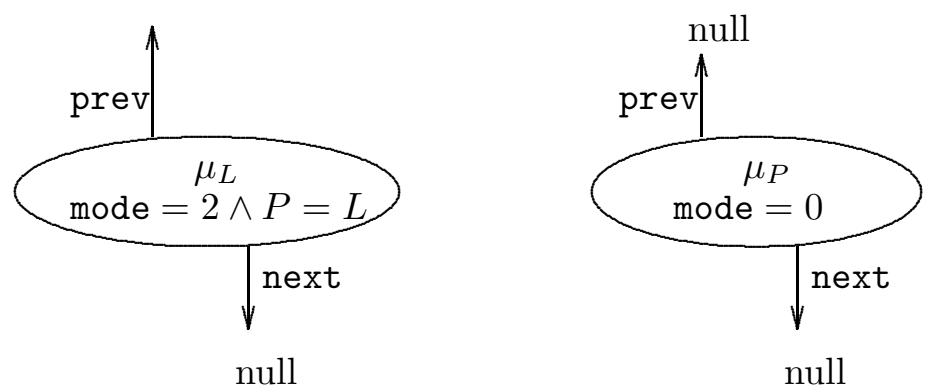

In step B, consistency among the chosen PDSI's has to be checked. For example, if $\mu_{L}$ requires that the local pointer next of PDSI $\mu_{L} \rightarrow$ prev does not point back to PDSI $\mu_{L}$, then we have to check whether this requirement is compatible with the recordings in $\mu_{L} \rightarrow$ prev.

After step C, the transition is executed, and the configuration among the chosen PDSI's changes to the following for the above-mentioned Modified MCS algorithm scenario.

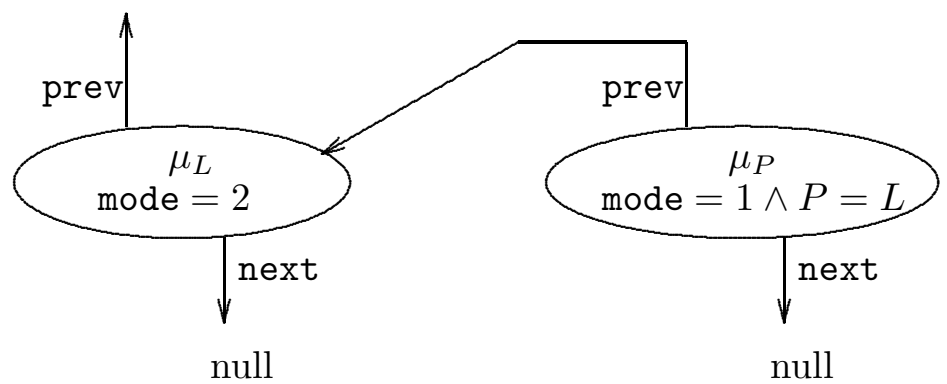

Till this step, there is no need to update $\chi_{A}$.

In step $D$, the propagation may go in three directions. First, we have to nondeterministically modify the reference information of those unchosen processes with references as one of the chosen processes. In this scenario, since global reference $L$ is reassigned to $\mu_{P}$, the global side-effect of this step is to update the global references of all PDSI's in $\chi_{A}$ so that every PDSI knows that $\mu_{P}$ has become the new $L$. Especially, we have to check if there is any PDSI $\bar{\mu}$ with $\bar{\mu} \rightarrow$ next points to the unique previously globally referenced $\mu_{L}$ and changes the recording of $\bar{\mu} \rightarrow$ next.

Second, we have to modify the ILM recordings of the references' of those chosen PDSI's if those chosen PDSI's are PSI-modified. In our scenario, we have to nondeterminstically pick a PDSI corresponding to $\mu_{L} \rightarrow$ prev and changes its ILM recordings. With the side-effect nondeterministically propagated, we assume that $\chi_{A}$ is changed to $\chi_{D}$ to reflect the propagation. 
Third, the new PDSI's of the chosens have to be collapsed with the PDSI's in $\chi_{D}$ respecting bound $B$. In this scenario, there will be two such PDSI's, one modified from $\mu_{P}$ (say $\mu_{P}^{\prime}$ ) and the other from $\mu_{L}$ (say $\mu_{L}^{\prime}$ ), to be added into $\chi_{D}$ again. In case that $\chi_{D}\left(\mu_{L}^{\prime}\right)=B$ or $\infty$, then $\chi_{D}\left(\mu_{L}^{\prime}\right)$ should be changed to $\infty$; otherwise, $\chi_{D}\left(\mu_{L}^{\prime}\right)$ is incremented by one.

In Step E, if and only if we found that $\chi_{D}$ is changed to be equal to $\chi^{\prime}$, we shall answer true. The performance of the procedure can be enhanced with symbolic manipulation techniques 6 , 8]. We are still investigating the details.

\section{Safety Bound Verfication with CIS}

The following lemma shows that CIS is indeed a conservative approximation of all reachable states from the initial states of all implementation.

Lemma 2. Given a concurrent algorithm $S$ with $X$ as the set of GDSI's of initial states of all implementations, the minimal set Reachable satisfying the following conditions:

- $X \subseteq$ Reachable; and

- for all $\chi \in$ Reachable and $\chi^{\prime} \in G D S I s e t$ such that $\mathbf{x t i o n}_{e}\left(\chi, \chi^{\prime}\right)=$ true for some transition rule $e, \chi^{\prime} \in$ Reachable.

is a super set of GDSI's of all reachable states of all implementaions of $S$.

Proof : Our GDSI overapproximates a global state by filtering out information over bound $B$. While nondeterministically computing $\operatorname{xtion}_{e}\left(\chi, \chi^{\prime}\right)$ for some GDSI's $\chi, \chi^{\prime}$ and transition rule $e$, overapproximation is also done by considering all possibilities with regard to the precision bound setup by $B$. Thus in the construction of Reachable, only overapproximation has been done and underapproximation is never done. Considering all these, we infer that Reachable is indeed a superset of GDSI's of reachable states of all implementaions of $S$.

By just naive enumerating all the GDSI's reachable from the initial state in an algorithm implementation, we will easily bump into combinatorial explosion of complexity because each PDSI can be mapped to any number in $[0, B]^{(\infty)}$. However, we can take advantage of our interleaving semantics to eliminate much of such complexity. The idea is based on lemma 3. A GDSI $\chi^{\prime}$ contains another GDSI $\chi$, in symbols $\chi \subseteq \chi^{\prime}$, if for every PDSI $\mu \in \operatorname{PDSIset}^{(B)}, \chi(\mu) \leq \chi^{\prime}(\mu)$.

Lemma 3. Suppose we have two GDSI's $\chi \subseteq \chi^{\prime}$. Then for every GDSI sequence $\chi_{0} \chi_{1} \ldots .$. with $\chi_{0}=\chi$, we can construct another GDSI sequence $\chi_{0}^{\prime} \chi_{1}^{\prime} \ldots .$. with $\chi_{0}^{\prime}=\chi^{\prime}$ such that for all $k \geq 0, \chi_{k} \subseteq \chi_{k}^{\prime}$ and $\chi_{k}$ and $\chi_{k}^{\prime}$ may go to $\chi_{k+1}$ and $\chi_{k+1}^{\prime}$ respectively with the same transition rule.

Proof : A pictorial explanation of this fact is in figure 3 The relation can happen because in a concurrent system without invariance conditions specified for the operation modes, we can withhold those PDSI's in $\chi^{\prime}$ but not in $\chi$ from firing transitions.

Now we shall present our approximation algorithm for safety bound problem with finite GDSI set as our CIS. We shall take advantage of lemma 3 such that two GDSI's $\chi, \chi^{\prime}$ will not be in the CIS simultaneously if $\chi \subseteq \chi^{\prime}$. 


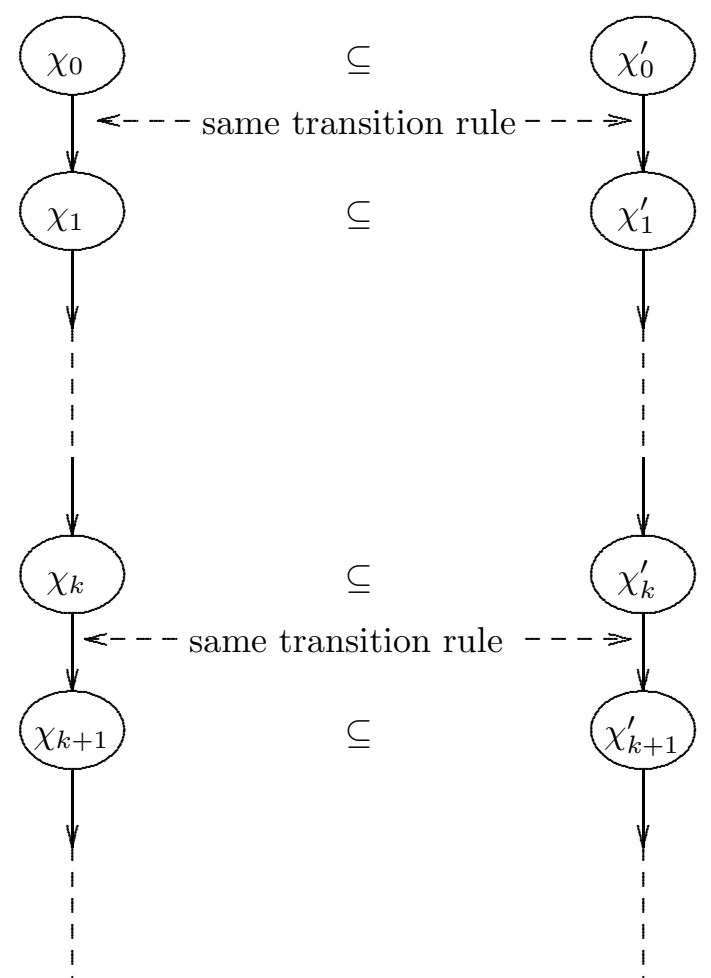

Fig. 3. Containing relation between GDSI sequences

Given a $\chi \in \operatorname{GDSIset}^{(B)}$, count $_{\text {pred }}(\chi)$ is also the number, respecting bound $B$, of PDSI's satisfying pred in $\chi$. Formally speaking,

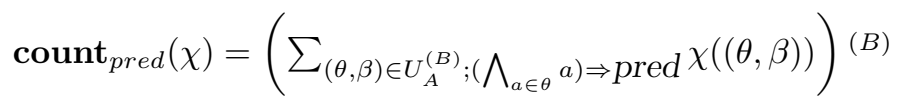

Now we have the procedure Safety_Bound() in table 1 to embody our saifety bound verification method in details. Note in statements (2.2.2) and (2.2.3), we delete those GDSI's contained by other GDSI's in $V$ according to lemma 3 .

The complexity of the method is polynomial to the number of GDSI's of states which then depends on $A(P)$ and $B$. A rough complexity analysis follows. The equivalence relation among pointers in a PDSI basically partitions global pointers, local pointers of $P$, the local pointers of references of $P$, the local pointers of references of those process pointed to by global pointers. The total number of pointers involved is $H=1+\left(\left|\operatorname{Ptr}_{g}\right|+\left|\operatorname{Ptr}_{l}\right|\right)\left(1+\left|\operatorname{Ptr}_{l}\right|\right)$ which is square to the size of $S$. The number of different partitions on these many pointers is roughly in the complexity of factorial to $H$. This will be the dominating factor in the complexity. Considering the values of $\operatorname{ILM}^{(B)}$, we can deduce that the number of different PDSI's is roughly $O\left((B+1)^{\mid \text {PSIset }_{\mid}} 2^{H}\right)=O\left(2^{|S|^{2}} \log B\right)$. Since GDSI 
$/^{*} S$ is an algorithm with transition rule set $E$.

$/^{*}$ pred is a process predicate describing the dangerous property.

$/^{*} C$ is the number of processes allowed in critical section.

$/^{*} B$ is the bound used in GDSI's. It is assumed $C \leq B$. */

Safety_Bound $(S$, pred, $C, B)\{$

(1) Generate the initial GDSI $\chi_{0}$; let $V:=\left\{\chi_{0}\right\} ; W:=V$;

(2) Repeat until $W=\emptyset$. \{

(1) If there is a $\chi \in V$ such that $\operatorname{count}_{\text {pred }}(\chi)>C$, report "don't know" and stop.

(2) Let $W:=\left\{\chi_{2} \mid \chi_{1} \in W ; \chi_{2} \in \operatorname{GDSIset}^{(B)} ; \exists e \in E\left(\operatorname{xtion}_{e}\left(\chi_{1}, \chi_{2}\right)\right)\right\}-V$;

(3) Let $V:=V \cup W$;

\}

(3) Report "SAFE!"

\}

Table 1. Safety analysis with CIS

are mappings from PDSI's to $[0, B]^{(\infty)}$, the total number of different GDSI's is then $(B+2)^{O\left(2^{|S|^{2} \log B}\right)}=2^{2^{O\left(|S|^{2} \log B+\log \log B\right)}}=2^{2^{O\left(|S|^{2} \log B\right)}}$. Thus our approach in each iteration of $B$ value is of complexity doubly exponential to $|S|^{2} \log B$.

For a lot of mutual exclusion protocols, small value of $B$ like 1 will work and the CIS's exhibit simple regularity. The complexity analyses for MCS mutualexclusion algorithm in section [ [ shall justify our claim.

\section{On Mellor-Crummy \& Scott's Algorithm}

We shall prove that our method indeed can verify Mellor-Crummy \& Scott's (MCS) locking algorithm 18 for mutual exclusion in concurrent systems. MCS locking algorithm is a provenly correct algorithm requiring little shared memory. We believe that our method can verify many such algorithms with small $B$ values regardless of the number of processes. The following lemma shows that our method can verify the modified version. A similar one can be used to prove for the original version.

Lemma 4. : In the CIS constructed for MCS locking algorithm as shown in figure 1 with $B=1$ and lemma , there is no image $\chi$ with count $_{\text {mode }=4}(\chi)>1$. Proof : A transition rule $e$ is executed by a process in exactly the same way as step $\mathrm{C}$ of $\mathbf{x t i o n}_{e}$ is operated on the corresponding PDSI's. Steps $A, B, D, E$ are nondeterministically performed to properly maintain the counts in the multisets and in the ILM's to consider all possibilities respecting the bound $B$. Only the transiting PDSI with the local locked $=$ true can make its-successor-in-thequeue's locked true. With $B=1$, we know in a GDSI whether there is only one PDSI with the local locked $=$ true. 
Intially, all PDSI's are in mode 0 with their local locked $=$ false. From that point on, we want to make sure that given two GDSI's $\chi, \chi^{\prime}$ and a transition rule $e$ with $\operatorname{xtion}_{e}\left(\chi, \chi^{\prime}\right)=$ true, if $\operatorname{count}_{\text {locked=true }}(\chi) \leq 1$, then $\operatorname{count}_{\text {locked }=\text { true }}\left(\chi^{\prime}\right) \leq 1$. The first PDSI detecting prev $=$ null while leaving mode 1 , will enter mode 4 with locked set to true. This is the only situation when we can go from $\operatorname{count}_{\text {locked }=\operatorname{true}}(\chi)=0$ to $\operatorname{count}_{\text {locked }=\operatorname{true}}\left(\chi^{\prime}\right)=1$.

According to the algorithm, only the PDSI with locked $=$ true can set the locked of other PDSI's to true. Immediately before setting the other PDSI's to true with transition rule

$$
\text { mode }=7 \rightarrow\left(\begin{array}{l}
\text { mode }:=0 ; \\
\text { locked }:=\text { false } \\
\text { next } \rightarrow \text { locked }:=\text { true } ; \\
\text { next }:=\text { null; }
\end{array}\right)
$$

the PDSI must first reset its own locked. With $B=1$, we know that count $_{\text {locked }=\text { true }}(\chi)$ and count $_{\text {locked }=\operatorname{true}}\left(\chi^{\prime}\right)$ will both be one. Considering both the two cases when a new PDSI has its local locked set to true, we find that count $_{\text {locked }=\text { true }}\left(\chi^{\prime}\right)=1$. We thus concluded that our CIS does not have a GDSI in which more than one PDSI's have their locked $=$ true.

We want to point out that our proof for lemma 4 is very much like a human proof for MCS algorithm. This shows that our method indeed reasons at an abstractness level similar to that of humans. Now we proceed to analyze the size of the CIS with $B=1$ for the modified MCS locking algorithm, in figure 1, which has the good property that once a process is in mode $=0$, its local pointers will all be set to null and no other processes will have local pointers pointing to it again. This make the data-structure pretty much "clean" without "stray" pointers. Thus the number of different GDSI's solely depends on the different possibilities of PDSI's near the queue head, noted by $H$, and tail as shown in figure 4. We thus have the following case analysis.

- In case there is only one PDSI in the queue. Then $H \rightarrow$ mode $\in\{1,4,5\}$ and there are 3 possibilities.

- In case there are two PDSI's in the queue. The values of $H \rightarrow$ next and $L \rightarrow$ prev depend on $H \rightarrow$ mode and $L \rightarrow$ mode. When $H \rightarrow$ mode $\in[1,6]$, $L \rightarrow$ mode $\in[1,3]$. When $H \rightarrow$ mode $=7, L \rightarrow$ mode $=3$. This accounts for $6 \times 3+1=19$ possibilities.

- In case there are three PDSI's in the queue. Similar to the reasoning in last item, we have $(6 \times 3+1) \times 3=57$ possibilities.

- In case there are more than three PDSI's in the queue. Assume the second PDSI in the queue is $H^{\prime}$ while the last second is $L^{\prime}$. The values of $H^{\prime} \rightarrow$ next and $L^{\prime} \rightarrow$ prev depends on the modes of the third and the last third PDSI's in the queue. Also the ILM's of $H^{\prime}$ and $L^{\prime}$ also depend on the third and the last third PDSI's in the queue. Moreover, all other PDSI's will be mapped to $\infty$ according to lemma 3 . Again, we have $(6 \times 3+1) \times 3 \times 3 \times 3 \times 3=1539$ possibilities.

Summing up all the possibilities in addition to the initial GDSI, we have $3+19+$ $57+1539+1=1619$ different GDSI's in our final CIS where the "1" represents the initial GDSI which maps the initial PDSI to $\infty$ and everything else to zero. 

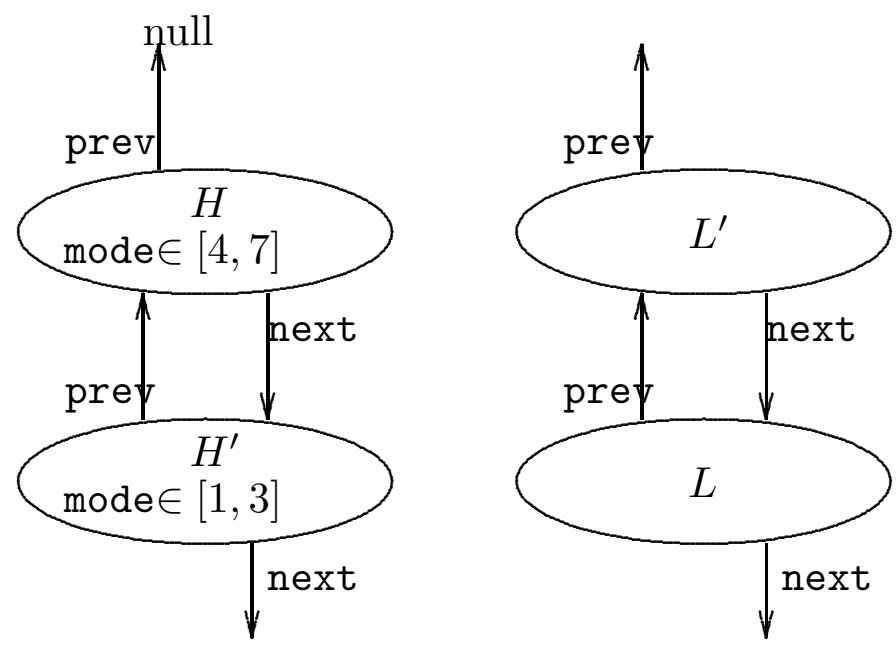

null

Fig. 4. PDSI pattern for modified MCS algorithm

\section{Conclusion}

With the known worst-case complexities of most verification problems in theory, it is apparent that the current technology of model-checking is incapable of verifying nontrivial software systems. We believe such a dilemma results from the fact that current verification theory does not distinguish "good" design from "bad" design. We argue our CIS technology is a successful example to verify welldesigned concurrent systems in which relations among different PDSI groups are more important than both the actuagl numbers of processes in each PDSI group and the actual values of all pointers. We feel hopeful our technology can be extended to verify well-designed concurrent systems with other types of infinite behaviors.

\section{Acknowledgment}

I sincerely thank the reviewers of FM'99 whose comments and suggestions have greatly improved the quality of the manuscript.

\section{References}

[1] R. Alur, C. Courcoubetis, D.L. Dill. Model Checkin in Dense Real-Time, Information and Computation 104, pp.2-34 (1993).

[2] R. Alur, T.A. Henzinger. Real-Time Logics: Complexity and Expressiveness. Information and Computation 104, pp.35-77 (1993). 
[3] K.R. Apt, D.C. Kozen. Limits for Automatic Verification on finite-state concurrent systems. Information Processing Letters, 22:307-309, 1986.

[4] F. Balarin. Approximate Reachability Analysis of Timed Automata. IEEE RTSS, 1996.

[5] M.C. Browne, E.M. Clarke, O. Grumberg. Reasoning about Networks with Many Identical Finite State Processes. Information and Computation 81, 13-31, 1989.

[6] J.R. Burch, E.M. Clarke, K.L. McMillan, D.L.Dill, L.J. Hwang. Symbolic Model Checking: $10^{20}$ States and Beyond, IEEE LICS, 1990.

[7] B. Boigelot, P. Godefroid. Symbolic Verification of Communication Protocols with Infinite State Spaces using QDDs. CAV 1996, LNCS, Springer-Verlag.

[8] R.E. Bryant. Graph-based Algorithms for Boolean Function Manipulation, IEEE Trans. Comput., C-35(8), 1986.

[9] E. Clarke and E.A. Emerson. Design and Synthesis of Synchronization Skeletons using Branching-Time Temporal Logic, Proceedings of Workshop on Logic of Programs, Lecture Notes in Computer Science 131, Springer-Verlag, 1981.

[10] E. Clarke, E.A. Emerson, and A.P. Sistla. Automatic Verification of Finite-State Concurrent Systems Using Temporal Logic Specifications, ACM Transactions on Programming Languages and Systems 8(2), 1986, pp. 244-263.

[11] E.M. Clarke, O. Grumberg, S. Jha. Verifying Parameterized Networks using Abstraction and Regular Languages. CONCUR'95, LNCS 962, Springer-Verlag.

[12] E.A. Emerson, K.S. Namjoshi. Reasoning about Rings. ACM POPL, 1995.

[13] E.A. Emerson, A.P. Sistla. Utilizing Symmetry when Model-Checking under Fairness Assumptions: An Automata-Theoretic Approach. ACM TOPLAS, Vol. 19, Nr. 4, July 1997, pp. 617-638.

[14] S.M. German, A.P. Sistla. Reasoning about Systems with Many Processes. Journal of ACM, Vol. 39, No. 3, July 1992, pp.675-735.

[15] J.E. Hopcroft, J.D. Ullman. Introduction to Automata Theory, Languages, and Computation, Addison-Wesley, 1979.

[16] R.P. Kurshan, K.L. McMillan. A Structural Induction Theorem for Processes. Information and Computation 117, 1-11(1995).

[17] D. Lesens, N. Halbwachs, P. Raymond. Automatic Verification of Parameterized Linear Networks of Processes. ACM POPL, 1997.

[18] J.M. Mellor-Crummey, M.L. Scott. "Algorithms for Scalable Synchronization on Shared-Memory Multiprocessors." ACM Transactions on Computer Systems, Vol. 9, No.1, Feb. 1991, pp.21-65.

[19] X. Nicolin, J. Sifakis, S. Yovine. Compiling real-time specifications into extended automata. IEEE TSE Special Issue on Real-Time Systems, Sept. 1992.

[20] F. Wang. Automatic Verification of Dynamic Linear Lists for All Number of Processes. Technical Report TR-IIS-98-019, Institute of Information Science, Academia Sinica, 1998.

[21] F. Wang, C.T. Lo. Procedure-Level Verification of Real-Time Concurrent Systems. Real-Time Systems Journal, Vol. 16, Nr. 1, Jan. 1999, Kluwer Academic Publishers. Preliminary version in Proceedings of the 1996 FME, Oxford, Britain, LNCS 1051, Springer-Verlag.

[22] F. Wang, A. Mok. RTL and Refutation by Positive Cycles, in Proceedings of the Formal Methods Europe Symposium, Barcelona, Spain, Octobor 1994, LNCS 873.

[23] F. Wang, A.K. Mok, E.A. Emerson. Real-Time Distributed System Specification and Verification in APTL. ACM TOSEM, Vol. 2, No. 4, Octobor 1993, pp. 346378.

[24] H. Wong-Toi. Symbolic Approximations for Verifying Real-Time Systems. Ph.D. thesis, Stanford University, 1995. 


\section{A Original MCS Locking Algorithm}

The modification follows good programming practice and can significantly reduce verification complexity. Two modifications in the modified MCS algorithm are

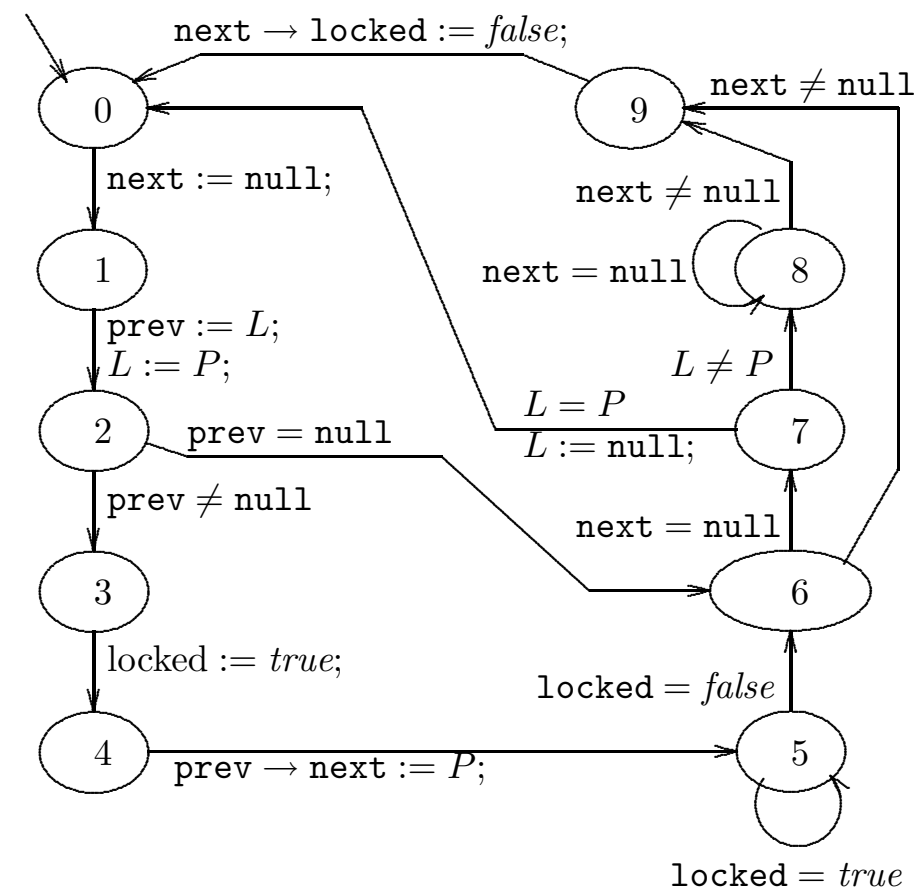

Fig. 5. original MCS locking algorithm

made. First, true and false for variable locked is interchanged in the modified version to be consistent with our initial state restrictions.

Second, local pointers are set to null as soon as the contents of the local pointers will not be used again. This is consistent with good programming practice. For example, in our modified algorithm, when a process releases the lock, it then also set its next to null because it is not meant in the queue already. However, in the original algorithm, this local next can still outdately point to some random process. Without cautious management, such "stray" local pointers can be mistakenly used. 